Document downloaded from:

http://hdl.handle.net/10251/180928

This paper must be cited as:

Salazar Afanador, A.; Safont, G.; Vergara Domínguez, L. (2019). A new method for fraud detection in credit cards based on transaction dynamics in subspaces. IEEE. 722-725. https://doi.org/10.1109/CSCl49370.2019.00137

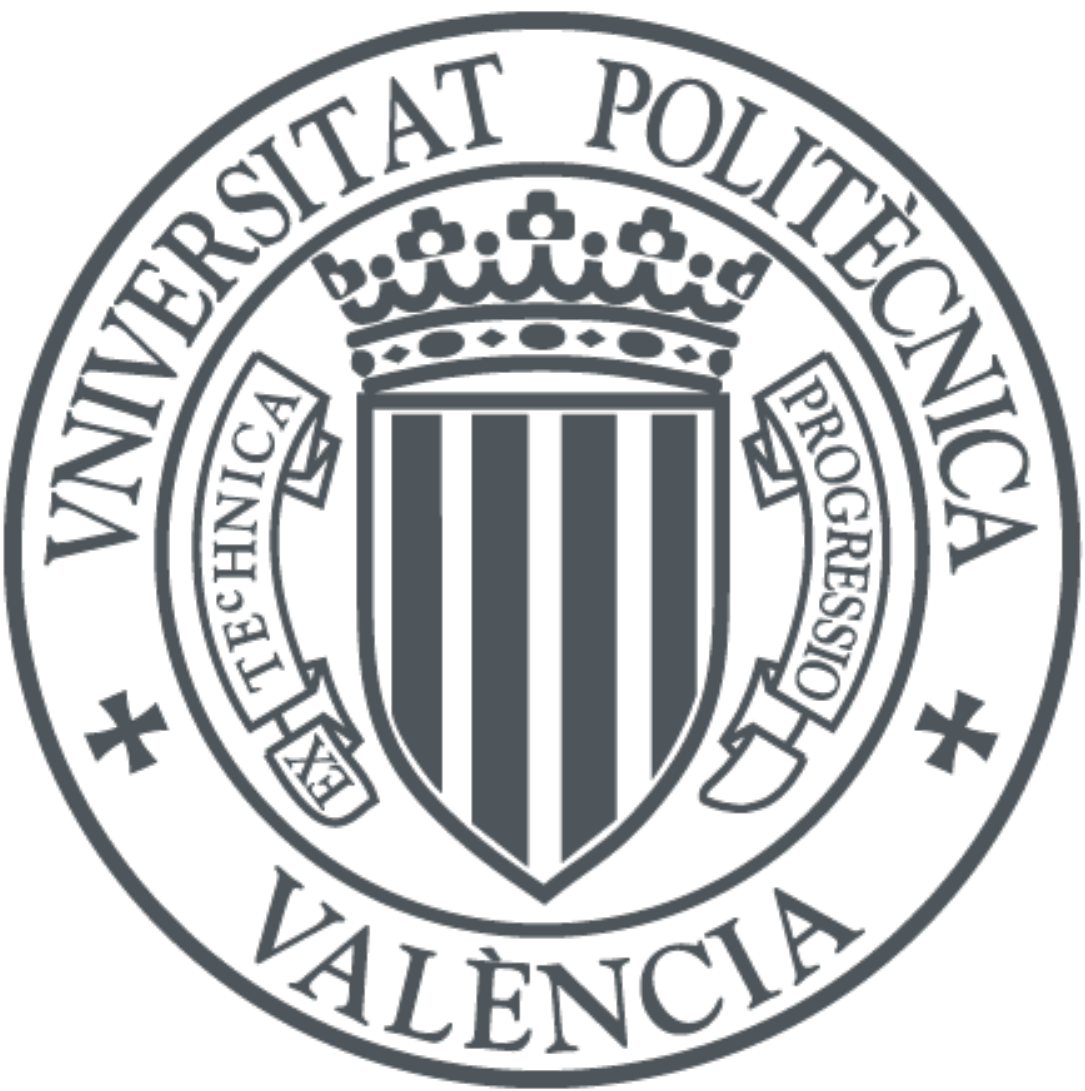

The final publication is available at

https://doi.org/10.1109/CSCI49370.2019.00137

Copyright IEEE

Additional Information 


\title{
A new method for fraud detection in credit cards based on transaction dynamics in subspaces
}

\author{
Addisson Salazar*, Gonzalo Safont, Luis Vergara \\ Institute of Telecommunications and Multimedia Applications, Universitat Politècnica de València, Valencia, Spain \\ asalazar@dcom.upv.es; gonsaar@upvnet.upv.es; lvergara@dcom.upv.es
}

\begin{abstract}
This paper presents a new method for fraud detection in credit cards based on exploiting the dynamics of the card transactions. We hypothesize different behavior models in the use of the card between legitimate clients and fraudsters that are registered in the sequential pattern that follows the transactions. The method considers analyses in subspaces defined by two or three variables recorded in the transactions. From these subspaces, several dynamic features, such as transaction velocity and acceleration, are estimated as input vectors for a classification process. Linear and quadratic discriminant analysis and random forest are implemented as single classifiers. All the single classification results obtained for each of the subspaces are late fused to obtain an overall result using alpha integration algorithm. The proposed method was evaluated using a subset of real data with a very low fraud to legitimate transaction ratio. We demonstrated that the temporal dependence of card transactions exploited in different subspaces and fused to give an overall result improves the detection accuracy of fraud detection in credit cards.
\end{abstract}

Keywords - classification, credit card fraud detection, decision fusion, transaction dynamics, alpha integration

Type of submission - Short Paper

Symposium-Signal \& Image Processing, Computer Vision \& Pattern Recognition (CSCI-ISPC)

\section{INTRODUCTION}

Fraud detection in credit card transactions is a challenging problem that affect financial companies, representing economical loss and degradation of customer perception of the company. There are several methods that have been proposed to solve this problem, see for instance [1-13] and the references within. The great effort of fraudsters for changing their strategies to attack fraud detection systems strongly affects the problem still remains open.

Most of the previous works on credit card fraud detection have used the whole set of variables of the card transaction record or have applied techniques of dimension reduction to decrease the number of variables to be processed. However, the variable space dimension remains high (e.g., from 10 to 40 variables). Instead, we will focus on experimental analyses in subspaces of low dimension processing subgroups of two or three variables from the card transaction record. In these subspaces, we will extract features with temporal dependence (dynamic features) searching for sequential patterns to distinguish between fraud and legitimate transactions. This tracking of transactions is done by card and thus the number of transactions in a month (typical time period for analysis) is low avoiding high dimensional analysis. Therefore, we approached the tracking of transactions in low dimension subspaces to make the problem tractable and understandable [14].

\footnotetext{
This work was supported by Generalitat Valenciana under grant PROMETEO/2019/109, and Spanish Administration and European Union grant TEC2017-84743-P.
}

The dynamic features are processed in each of the subspaces using three single classifiers: linear and quadratic discriminant analysis (LDA and QDA) and random forest (RDF). The proposed method also includes two steps of decision fusion of the results provided by the single classifiers [15-17]. The first decision fusion consist of combining the results of the classifiers at subspace level, i.e., obtaining a fused result for each of the subspaces. The second decision fusion consists of obtaining an overall result by combining all the fused results obtained for each of the subspaces. We applied the alpha integration technique to fuse the scores (posterior probabilities) given to transactions by the single classifiers [18-21]. This is called "late fusion", in contrast with "early fusion" that is the fusion of features made before classification [17]. Alpha integration provides weights for an optimum linear combination of the scores with respect to criteria such as least mean squares (LMSE) or the minimum probability of error (MPE) [22-24].

The following sections are defined as follows. Section 2 explains the proposed method for detection of frauds in credit card transactions. Section 3 describes the results obtained for a subset of real data from an international financial company. Finally, the conclusions and future work are included in Section 4.

\section{Proposed Method}

Fig. 1 shows an outline of the different steps of processing followed by the proposed method. 2D and 3D subspaces were defined selecting variables of the card transaction record with the help of experts. We were looking for variables with dissimilar temporal patterns between legitimate and fraud transactions. For instance, most of the cards are used in only one country, so transactions of the same card in different countries within a very short temporal gap should be suspicious. Another example is the relationship between amount and velocity of transactions. Frequently, fraud transactions are progressively increasing both amount and velocity between transactions; and conversely, legitimate transactions do not usually follow that kind of temporal pattern. Thus, six variables were selected to form different 2D and 3D variable combinations, i.e., subspaces for analysis.

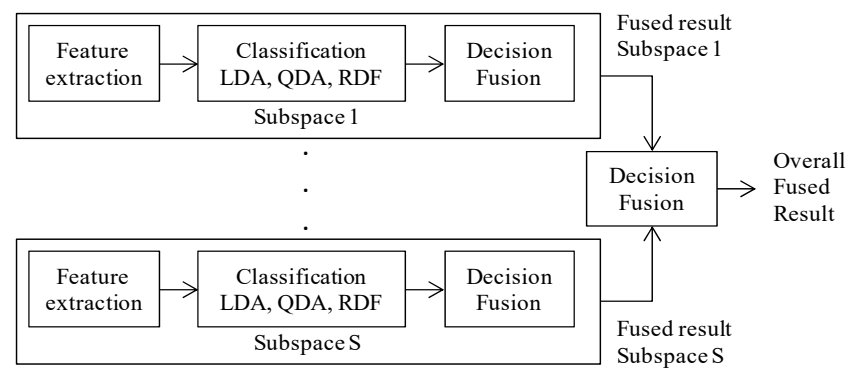

Fig. 1. Diagram of the proposed method. 
Among those variables were: amount, transaction velocity, and country changes of the transaction.

The analyzed data consisted of a subset of $1,762,374$ transactions from an international financial company

a)

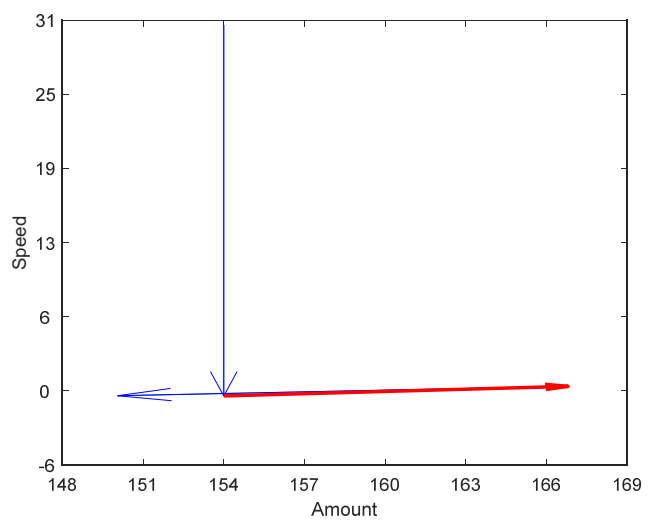

b)

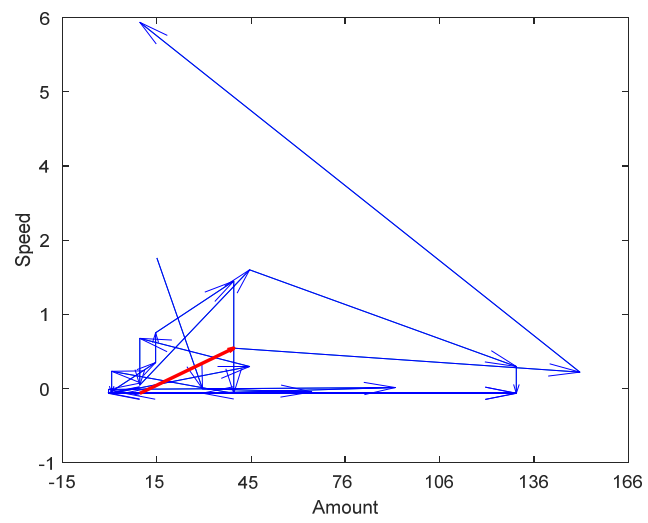

Fig. 2. Examples of the behavior of the transactions of a card in 2D subspaces.

a)

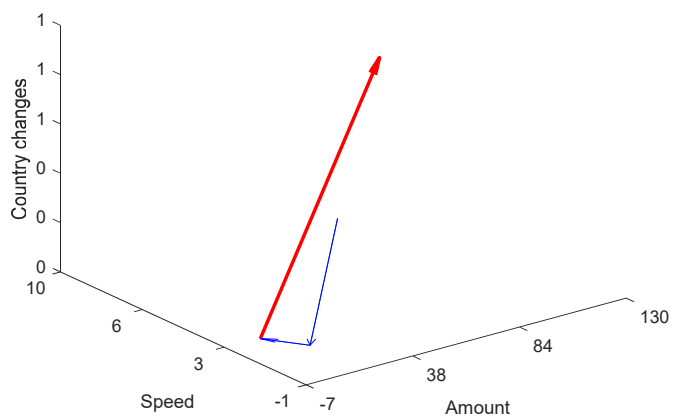

b)

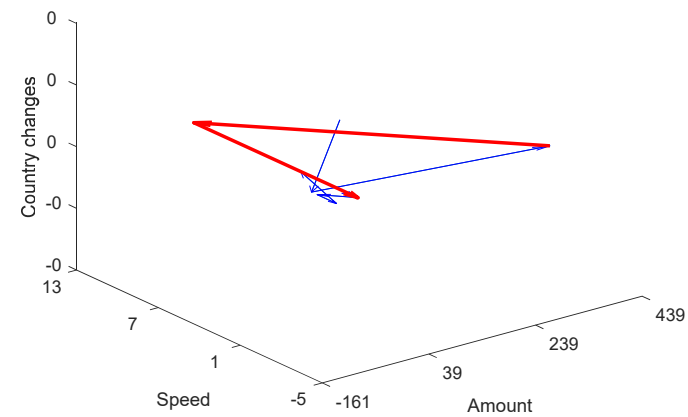

Fig. 3. Examples of the behavior of the transactions of a card in $3 \mathrm{D}$ subspaces. corresponding to 148,200 cards with $0.22 \%$ of the transactions were frauds.

Fig. 2 and Fig. 3 shows some examples of the behavior of the transactions of a card in 2D and 3D subspaces, respectively. Those figures are directed graphs that show temporal patterns following the arrowheads. Blue and red arrows correspond to legitimate and fraud transactions, respectively. It can be seen patterns are more distinguishable in Fig. 2.a and Fig. 3.a.

\section{A. Feature extraction}

In order to exploit the temporal patterns that are observable in the subspaces, the following features sensitive to changes in the transaction dynamics were estimated: mean; variance; mean axial velocity; number of changes in speed sign; total mean velocity; and rotation angle between the variables.

\section{B. Alpha integration information fusion technique}

Alpha integration was first proposed for the binary classification (detection) problem [18]. Let us assume that we have a group of $D$ binary classifiers (detectors) working on the detection problem. Each detector will produce a score $s_{i}, i=$ $1 \ldots D$, where higher values of $s_{i}$ indicate that the positive class is more likely than the negative class. In this context, alpha integration performs the optimal integration of these scores $\mathbf{S}=\left[\begin{array}{lll}s_{1} & \ldots & s_{D}\end{array}\right]^{T}$ into a single score $s_{\alpha}$ such that

$$
s_{\alpha}(\mathbf{s})=\left\{\begin{array}{cc}
{\left[\sum_{i=1}^{D} w_{i}\left(s_{i}\right)^{(1-\alpha) / 2}\right]^{2 /(1-\alpha)}} & , \alpha \neq 1 \\
\exp \left[\sum_{i=1}^{D} w_{i} \log \left(s_{i}\right)\right] & , \alpha=1
\end{array}\right.
$$

where $\alpha$ and the coefficients $\mathbf{w}=\left[w_{1} \ldots w_{D}\right]^{T}$ are the parameters to be optimized, subject to $w_{i} \geq 0, \sum_{i=1}^{D} w_{i}=1$. Due to these constraints, $s_{\alpha}$ is bound between 0 and 1 . It can be shown that many classical late soft fusion techniques are particular cases of alpha integration, such as the average ( $\alpha=$ -1 and $\left.w_{i}=1 / D \forall D\right)$, the minimum $(\alpha=\infty)$ and the maximum $(\alpha=-\infty)$. In practice, there are many applications where the parameters of alpha integration are unknown beforehand and have to be estimated from some training data. Previous works have presented the derivations required to optimize alpha integration with respect to the least mean squares (LMSE) and the minimum probability of error (MPE) criteria [22-23].

\section{EXPERIMENTAL RESULTS}

Fig. 4 and Fig. 5 show the results corresponding to the application of the method proposed in Section 2 for 2D and 3D subspaces, respectively. For clarity, we only show the results of the first decision fusion of the classifiers for each subspace, and the result of the second (overall) decision fusion. It can be seen that the overall decision fusion of the results for all the subspaces improve the results of fraud detection in terms of receiver operating characteristic (ROC) curve in the full range of values of low and very low false alarm (from 0 to $10 \%$ ). Note that this tight range of false alarm is the range of interest for this application, considering the economic and corporate reputation costs involved.

Fig. 6 shows a summary of the results that includes the overall fusion results of processing in 2D and 3D subspaces; the results obtained by the single classifiers using the whole space of variables of the card transaction record; and the fusion of those classifiers. Clearly, the results using the whole set of the available variables without extracting dynamic 


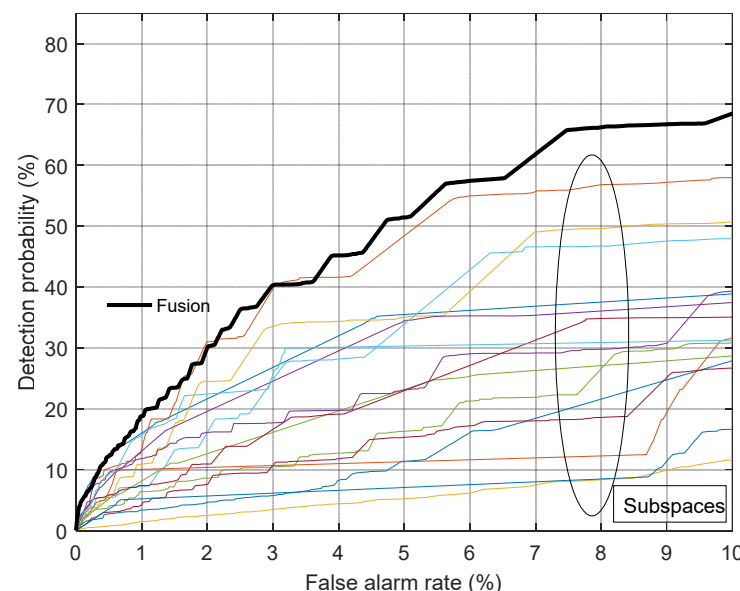

Fig. 4. Results of the experiment of the proposed method on 2D subspaces.

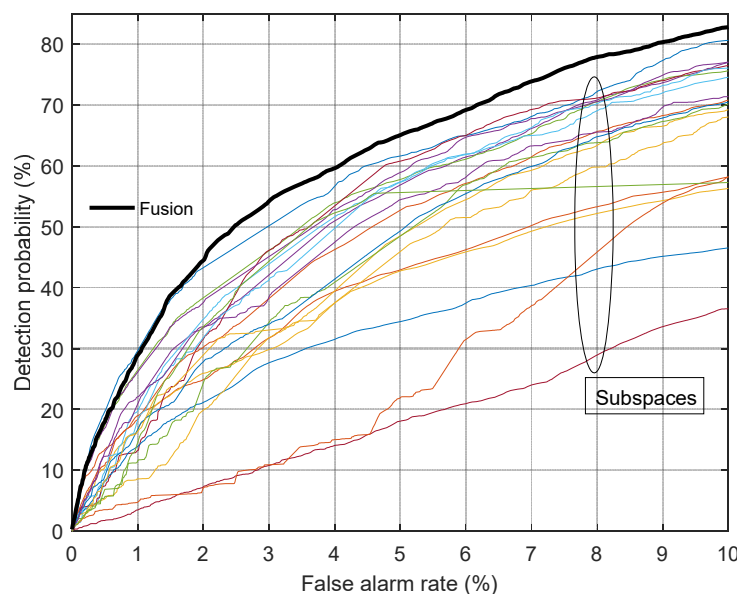

Fig. 5. Results of the experiment of the proposed method on 3D subspaces.

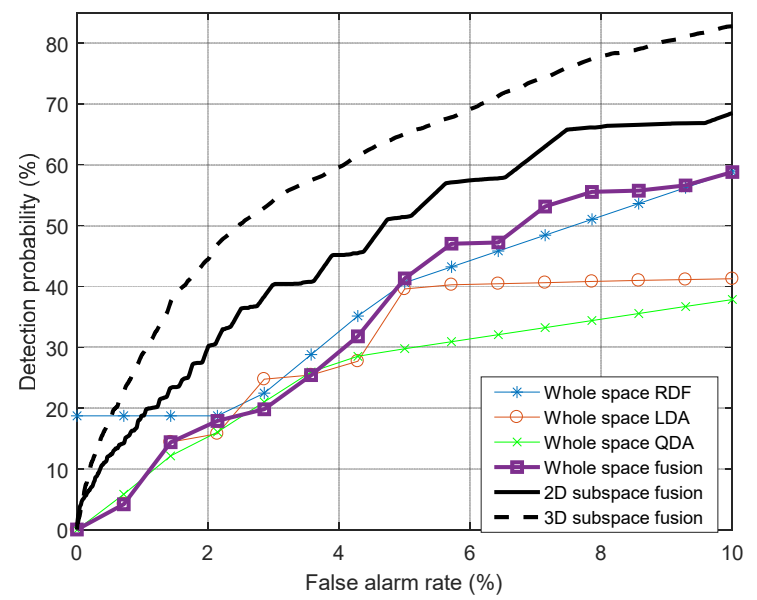

Fig. 6. Results of the experiment when considering the whole set of features, compared with the fused results for $2 \mathrm{D}$ and $3 \mathrm{D}$ subspaces.

features is worse than the results obtained by the proposed method. Furthermore, the fusion of 3D subspaces yielded a better result than the fusion of $2 \mathrm{D}$ subspaces.

The improvement of results also can be quantified estimating the area under the curve (AUC) for each of the ROC curves of Fig. 6 . This is shown in Table I.
TABLE I. AREA UNDER THE CURVE VALUES FOR THE RESULTS SHOWN IN FIG. 6

\begin{tabular}{|c|c|}
\hline Method & AUC \\
\hline RDF & 37.1753 \\
\hline LDA & 29.7744 \\
\hline QDA & 25.7966 \\
\hline Whole space fusion & 35.6832 \\
\hline Proposed 2D subspace fusion & 47.2444 \\
\hline Proposed 3D subspace fusion & 59.9476 \\
\hline
\end{tabular}

\section{CONCLUSIONS}

A new method for detection of frauds in credit card transactions has been presented. The method is intended to exploit sequential or temporal patterns using dynamic features in low dimension subspaces of two and three variables using three classifiers in each of the subspaces. This causes the production of multiple solutions and results to the problem.

An advanced decision fusion method called alpha integration is applied in two steps. In the first step, alpha integration obtains a single result for each $2 \mathrm{D}$ or $3 \mathrm{D}$ subspace. In the second step, the result for each subspace is fused to obtain the overall fused result, i.e., the global result. Results show the improvement of detection performance of the proposed subspace processing method compared with the results by processing the whole set of variables, without extraction of dynamic features. Thus, it was confirmed that the temporal patterns in the transactions in low dimension subspaces were able to distinguish the behavior of fraudsters from that of legitimate customers. 3D subspaces yielded a better result than 2D subspaces. This suggests that the optimal dimension of the subspaces might potentially be larger than 3 variables. Thus, there is a need to determine the optimal dimension and subspace structure that enable to separate between the two classes of credit card transactions. In any case, it would be constrained by the ratio of the number of variables to the number of records available.

Future works will focus on three topics. Firstly, this work has been completely experimental. Thus, future works will derive the theoretical basis for the proposed method and thoroughly demonstrate the results with an extensive number of experiments. Secondly, semi-supervised training will be considered to better exploit the large amount of transactions available $[25,26]$. Thirdly, the segmentation of the whole space into subspaces that are considered independently is similar to independent component analysis [27-29], which transforms the input variables into a set of independent components. However, the subspaces in this work was decided with the help of experts. Future works will consider hierarchical and knowledge discovery to automatically determine the dimension and composition of the optimal subspaces [30-33].

\section{REFERENCES}

[1] M. Ahmeda, A.N. Mahmooda, and R. Islam, "A survey of anomaly detection techniques in financial domain," Future Generation Computer Systems, vol. 55, pp. 278-288, 2016.

[2] S. Bhattacharyya, S. Jha, K. Tharakunnel, and J.C. Westland, "Data mining for credit card fraud: A comparative study," Decision Support Systems, vol. 50, pp. 602-613, 2011. 
[3] R.J. Bolton and D.J. Han, "Statistical fraud detection: A review," Statistical Science, vol. 17, no. 3, pp. 235-255, 2002.

[4] S. Panigrahi, A. Kundu, S. Sural, and A.K. Majumdar, "Credit card fraud detection: A fusion approach using Dempster-Shafer theory and Bayesian learning," Information Fusion, vol. 10, pp. 354-363, 2009.

[5] C. Phua, V. Lee, K. Smith, and R. Gayler, "A comprehensive survey of data mining-based fraud detection research," Computer Research Repository, pp. 1-14, 2010.

[6] S.B.E. Raj and A.A Portia, "Analysis on credit card fraud detection methods," in IEEE International Conference on Computer, Communication and Electrical Technology - ICCCET2011, Tamilnadu, India, 2011, pp. 152-156.

[7] P. Wongchinsri and W. Kuratach, "A survey - Data mining frameworks in credit card processing," in 13th International Conference on Electrical Engineering/Electronics, Computer, Telecommunications and Information Technology, ECTI-CON 2016, Chiang Mai, Thailand, 2016, pp. 1-6.

[8] A. Salazar, G. Safont, A. Soriano, and L. Vergara, "Automatic credit card fraud detection based on non-linear signal processing," proceedings of International Carnahan Conference on Security Technology (ICCST), pp. 207-212, Boston, MA (USA), 2012.

[9] A. Salazar, G. Safont, and L. Vergara, "Surrogate techniques for testing fraud detection algorithms in credit card operations," proceedings of International Carnahan Conference on Security Technology (ICCST), pp. 1-6, Rome (Italy), 2014.

[10] L. Vergara, A. Salazar, J. Belda, G. Safont, S. Moral, and S. Iglesias, "Signal processing on graphs for improving automatic credit card fraud detection," in proceedings of International Carnahan Conference on Security Technology (ICCST), pp. 1-6, Madrid (Spain), 2017.

[11] A. Salazar, G. Safont, A. Rodriguez, and L. Vergara, "Combination of multiple detectors for credit card fraud detection," in proceedings of International Symposium on Signal Processing and Information Technology (ISSPIT), pp. 138-143, Limassol (Cyprus), 2016.

[12] A. Salazar, G. Safont, and L. Vergara, "Semi-supervised learning for imbalanced classification of credit card transaction," in proceedings of International Joint Conference on Neural Networks (IJCNN), pp. 1-7, Rio de Janeiro (Brazil), 2018.

[13] A. Salazar, G. Safont, A. Rodriguez, and L. Vergara "New Perspectives of Pattern Recognition for Automatic Credit Card Fraud Detection," in Encyclopedia of Information Science and Technology, Fourth Edition, pp. 4937-4950, IGI Global, 2018.

[14] Séverine Dubuisson. Tracking with Particle Filter for Highdimensional observation and State Spaces. Digital Signal and Image Processing Series. Wiley, 2015.

[15] S. Yuksel, J. Wilson, and P. Gader, "Twenty years of mixture of experts," IEEE Transactions on Neural Networks and Learning Systems, vol. 23, pp. 1177-1193, 2012.

[16] B. Khaleghi, A. Khamis, F. Karray, and S. Razavi, "Multisensor data fusion: A review of the state-of-the-art," Information Fusion, vol. 14, pp. 28-44, 2013.

[17] M. Mohandes, M. Deriche, and S. Aliyu, "Classifiers Combination Techniques: A Comprehensive Review," IEEE Access, vol. 6, pp. 19626-19639, 2018.
[18] S. Amari, "Integration of stochastic models by minimizing $\alpha$ divergence," Neural Computation, vol. 19, 2796-2780, 2007.

[19] H. Choi, S. Choi, A. Katake, and Y. Choe, "Learning $\alpha$-integration with partially labeled data," in proceedings of IEEE International Conference on Acoustics, Speech, and Signal Processing (ICASSP), pp. 2058-2061, Piscataway, NJ (USA), 2010.

[20] H. Choi, S. Choi, and Y. Choe, "Parameter Learning for Alpha Integration," Neural Computation, vol. 25, pp. 1585-1604, 2013.

[21] D. Wu, "Parameter estimation for $\alpha$-GMM based on maximum likelihood criterion," Neural Computation, vol. 21, pp. 1776-1795, 2009.

[22] A. Soriano, L. Vergara, A. Bouziane, and A. Salazar, "Fusion of scores in a detection context based on alpha-integration," Neural Computation, vol. 27, 1983-2010, 2015.

[23] G. Safont, A. Salazar, and L. Vergara, "Multiclass alpha integration of scores from multiple classifiers," Neural Computation, vol. 31, no. 4 , pp. 806-825, 2019.

[24] S. Amari, Information Geometry and its Applications. Springer, 2016.

[25] J. Igual, A. Salazar, G. Safont, and L. Vergara, "Semi-supervised Bayesian classification of materials with impact-echo signals," Sensors, vol. 15, no. 5, pp. 11528-11550, 2015.

[26] A. Salazar, L. Vergara, "ICA mixtures applied to ultrasonic nondestructive classification of archaeological ceramics," EURASIP Journal on Advances in Signal Processing, vol. 2010, art. no. 8, 2010.

[27] R. Llinares, J. Igual, A, Salazar, and A. Camacho, "Semi-blind source extraction of atrial activity by combining statistical and spectral features," Digital Signal Processing: A Review Journal, vol. 21, no. 2, pp. 391-403, 2011.

[28] G. Safont, A. Salazar, A. Rodriguez, and L. Vergara, "On Recovering missing ground penetrating radar traces by statistical interpolation methods," Remote Sensing, vol. 6, no. 8, pp. 7546-7565, 2014.

[29] G. Safont, A. Salazar, L. Vergara, E. Gomez, and V. Villanueva, "Probabilistic Distance for Mixtures of Independent Component Analyzers, “ IEEE Transactions on Neural Networks and Learning Systems, vol. 29, no. 4, pp. 1161-1173, 2018.

[30] O. Maimon, and L. Rokach, Data mining and knowledge discovery handbook. Springer, 2005.

[31] A. Salazar, J. Gosalbez, I. Bosch, R. Miralles, and L. Vergara "A case study of knowledge discovery on academic achievement, student desertion and student retention," in proceedings of the 2nd International Conference on Information Technology: Research and Education (ITRE), pp. 150-154, London (UK), 2004.

[32] A. Salazar, J. Igual, L. Vergara, and A. Serrano, "Learning hierarchies from ICA mixtures," in proceedings of theIEEE International Joint Conference on Artificial Neural Networks, pp. 2271-2276, 2007.

[33] A. Salazar, J. Igual, G. Safont, L. Vergara, and A. Vidal, "Image applications of agglomerative clustering using mixtures of nonGaussian distributions," in proceedings of the International Conference on Computational Science and Computational Intelligence (CSCI), pp. 459-463, Las Vegas, NS (USA), 2015. 